\title{
La normativa de atribución de rentas a establecimientos permanentes en Colombia
}

\section{Attribution of Profits to Permanent Establishments in Colombian Tax Law}

\author{
AITOR NAVARRo IBARROLA ${ }^{1}$
}

\section{Resumen}

En el presente trabajo se analizan las reglas para la determinación de la renta de establecimientos permanentes situados en Colombia. A tal efecto, el autor lleva a cabo un estudio sobre la base de las pautas que la OCDE establece en la materia, ya que el sistema de conceptos propuesto por este organismo internacional se adoptó como referencia por parte del legislador colombiano a partir de 2012. En concreto, en el marco del concepto de entidad funcionalmente separada, se analiza el contenido posible de las funciones, activos y riesgos susceptibles de ser asignados a un EP, así como las operaciones internas que lleva a cabo con la casa central y terceros. Cabe destacar así mismo que las referidas pautas están siendo objeto de revisión en el marco del proyecto BEPS, modificaciones que podrían tener impacto en la interpretación de la norma interna colombiana.
Palabras clave: Impuesto sobre la renta; Establecimiento permanente; Atribución de rentas; Entidad funcionalmente separada; Authorized OECD Approach; BEPS.

\section{Abstract}

In this contribution, the rules on attribution of profits to permanent establishments enacted in Colombian tax law are analyzed. To that effect, the author develops a study on the grounds determined by the OECD in the subject matter, as the framework of concepts posed by this institution was adopted in Colombian legislator since 2012. In particular, the functionally separate entity notion was studied alongside the concepts of functions, risks and assets as factors likely to be attributed to a PE, as well as the dealings that a PE conduct with its head office and third parties. It is worth mentioning that the standards proposed by the OECD are being subject to further modifications within the BEPS project,

${ }^{1}$ Abogado, Doctor en Derecho de la Universidad Carlos III de Madrid. Profesor de Derecho Financiero y Tributario en la Universidad Carlos III de Madrid. España, Madrid. correo-e: aitor. navarro@uc3m.es.Artículo recibido el 1 de agosto de 2016. Aprobado el 20 de septiembre de 2016. Para citar el artículo: Navarro,A. (2016). "La normativa de atribución de rentas a establecimientos permanentes en Colombia", en Revista de Derecho Fiscal n 9, Bogotá: Universidad Externado de Colombia. pp. 43-62. DOI: http://dx.doi.org/10.18601/16926722.n9.04 
changes that may impact the interpretation of Colombian domestic rules on the subject.

Keywords: Colombian Income Tax; Permanent Establishment; Attribution of Income; Functionally Separate Entity; Authorized OECD Approach; BEPS.

Sumario: I. Introducción. II. Aproximación al concepto de entidad funcionalmente separada y su implementación en el ordenamiento jurídico tributario colombiano. III. Análisis de las reglas de atribución de beneficios a establecimientos permanentes en la normativa colombiana. 1. Primera fase: conformación de una entidad funcionalmente separada. 1.1. La atribución de funciones como punto de partida del análisis. 1.2. La atribución de activos al EP de acuerdo al concepto de propiedad económica. 1.3. Especialidades en la atribución de fuentes de financiación al EP 1.4. La asignación de riesgos al EP como entidad funcionalmente separada. 2. Segunda fase: atribución de ingresos y gastos derivados de acuerdos entre la casa central y el EP así como con terceras entidades. 3. La relevancia de la contabilidad en la atribución de rentas a EP. IV. Conclusiones. V. Referencias.

\section{Introducción}

La tributación de rentas empresariales en el ámbito internacional se basa en el principio del beneficio, según el cual si un no residente supera un cierto nivel de presencia física en un Estado, se considera que existe un vínculo suficiente (economic allegiance) que legitima el gravamen de las rentas derivadas de su actividad, en tanto el contribuyente disfruta de los servicios que dicho Estado provee en su territorio ${ }^{2}$. Desde el consenso alcanzado por la comunidad internacional en los años veinte del siglo pasado, se emplea la noción de establecimiento permanente (EP en adelante), generalmente caracterizado como un lugar fijo de negocios ${ }^{3}$, para concretar ese grado de vinculación al que hacemos referencia. Este planteamiento encuentra reflejo en la mayoría de los convenios para evitar la doble imposición (CDI en adelante) en vigor a nivel mundial ${ }^{4}$. Además, la normativa interna de un gran número de Estados emplea la figura del EP para asimilar el tratamiento tributario de las rentas que le son imputables al de las sociedades residentes.

En este contexto, la correcta atribución de las rentas al EP es crucial para la justa cuantificación de la carga impositiva que una empresa no residente debe soportar en el país en que el EP se sitúa ${ }^{5}$ Aunque existen diversas fórmulas al efecto, la más extendida consiste en tratar al EP como si fuese una entidad funcional separada e independiente de la casa central. Esta ficción trata de emular el principio de empresa separada que rige la imposición sobre sociedades

\footnotetext{
${ }^{2}$ Vid. Skaar (1991, pp. 27-29); Escribano López (2015, pp. 7-8).

${ }^{3}$ Vid. la definición contenida en el artículo 20-1 del Estatuto Tributario según redacción dada por la Ley 1607 de 2012 en el art. 5 del Modelo de Convenio OCDE (MCOCDE en adelante), el art. 5 del Modelo de Naciones Unidas (MONU en adelante) y el art. 5 del Modelo de Convenio de Estados Unidos (MCEEUU en adelante).

${ }^{4}$ Los modelos de Convenio OCDE, ONU y EE.UU. recogen este esquema en el precepto dedicado a la distribución de la potestad tributaria de rentas empresariales. Vid. art. 7.2 MCOCDE-2014, art. 7.2 MCONU-2011, art. 7.2 MCEEUU-2016.

${ }^{5}$ Cuando nos referimos a la correcta atribución de rentas, la expresión comprende tanto la asignación de beneficios como de pérdidas. Vid. en el mismo sentido, OCDE (2010) Report on the Attribution of Profits to Permanent Establishments, par. 3.
} 
desde la aparición de los sistemas tributarios modernos a principios del siglo XX.

El objeto del presente es analizar las reglas de atribución de beneficios a establecimientos permanentes presentes en la normativa colombiana. En Colombia, desde la reforma operada por la Ley 1607 de 2012, se ha adoptado un sistema de conceptos idéntico al que la OCDE propone ${ }^{6}$. La necesidad de concretar su contenido y la existencia de pautas elaboradas por dicha institución internacional en la materia son los factores más importantes que sustentan la relevancia de acometer un estudio sobre la cuestión apuntada.

La estructura del presente trabajo consta de dos partes. En la primera ahondaremos en el significado de la noción de entidad funcionalmente separada y el contenido específico de la normativa colombiana como punto de partida para la interpretación de la normativa sobre atribución de beneficios (apartado II). En la segunda, analizaremos en profundidad el conjunto de normas presentes en el Estatuto Tributario y la normativa reglamentaria de desarrollo para atribuir beneficios a EP (apartado III), distinguiendo entre la primera fase, de atribución de funciones, activos y riesgos (apartado A); la segunda fase, relativa al estudio de las operaciones realizadas por parte del EP con su casa central y con ter- ceros (apartado B), para finalizar resaltando la importancia de la correcta documentación del análisis (apartado C). Finaliza la exposición con una breve conclusión del estudio realizado (apartado IV).

\section{Aproximación al concepto de entidad funcionalmente separada y su implementación en el ordenamiento jurídico tributario colombiano}

En el ámbito de la fiscalidad internacional, el concepto de empresa separada es el pilar fundamental sobre el que se asienta la atribución de beneficios a un EP. Desde 1933 se viene considerando que un EP debería ser considerado y tratado como una empresa independiente ${ }^{7}$. De esta forma, la renta gravable debía basarse en la existencia del EP como ente separado de la casa central ${ }^{8}$. Así se logra gravar únicamente el beneficio neto que se origina en el territorio donde el EP se sitúa, con independencia del resultado general de la empresa9 ${ }^{9}$. Tal aproximación implica, en buena lógica, tratar al EP como si fuese una empresa distinta y separada, que tendría capacidad para concluir acuerdos no solo con entes terceros o vinculados, sino también con la casa central ${ }^{10}$. En este sentido, es habitual el uso de la expresión "entidad funcionalmen-

\footnotetext{
${ }^{6}$ OCDE (1994). Issues in International Taxation No. 5: Model Tax Convention: Attribution of Income to Permanent Establishments. OCDE (2001). Discussion Draft on the Attribution of Profits to Permanent Establishments. OCDE (2004). Discussion Draft on the Attribution of profits to permanent establishments. OCDE (2006). Report on the Attribution of Profits to Permanent Establishments. OCDE (2008). Report on the Attribution of Profits to Permanent Establishments. OCDE (2010). Report on the Attribution of Profits to Permanent Establishments.

${ }^{7}$ Vid. los trabajos de Mitchell Carroll y Ralph Jones en la materia: Carroll y Jones (1932-1933) Taxation of Foreign and National Enterprises, vols. 1-5 (Liga de las Naciones). Carroll, M. (1933) Volume 4. Methods of Allocating Taxable Income, (Liga de las Naciones) y Jones, R. (1933) Volume 5 Allocation Accounting for the Taxable Income of Industrial Enterprises (Liga de las Naciones). Carroll en su momento recomendó que "la asignación de renta a una sucursal debería ser equivalente a la que se hubiese atribuido a una entidad independiente". Vid. Vann (2006, p. 364). ${ }^{8}$ El concepto de empresa separada no ha variado desde el año de su formulación. Vid. Sasseville y Vann (2016, p. 37).

${ }_{9}^{9}$ García Prats (1996, p. 263).

${ }^{10}$ Bennet y Russo (2009, p. 76).
} 
te separada" (functionally separate entity) ${ }^{11}$ para señalar el grado de independencia del EP con respecto al resto de la empresa, de manera que una empresa podría sufrir pérdidas en términos agregados y sin embargo obtener beneficios a nivel de EP, beneficios que serían gravados por el Estado de localización del $\mathrm{EP}^{12}$.

El principio de empresa separada es un mandato demasiado genérico, que es necesario concretar. A tal efecto, a lo largo de los años, la OCDE ha publicado una serie de pautas que muchos Estados han seguido a la hora no solo de interpretar y aplicar el artículo sobre beneficios empresariales de su red de CDI, sino que también se ha empleado como modelo para establecer reglas para la atribución de beneficios a EP en Derecho interno, como ha sucedido recientemente en Colombia.

La metodología para la asignación de beneficios propuesta por la OCDE ha evolucionado del mero análisis de la contabilidad y demás documentos internos de la empresa hacia un estudio de las funciones, activos y riesgos atribuibles al $\mathrm{EP}$, elementos que en última instancia definirán ese carácter de entidad autónoma e independiente que la noción de entidad funcionalmente separada pretende lograr, de acuerdo con las características económicas relevantes (economically relevant characteristics) del $\mathrm{EP}^{13}$. A este análisis se añade una segunda fase, consistente en determinar los ingresos y gastos derivados de los acuerdos del EP con la casa central, con otros EP, con entidades vinculadas y con terceros en atención a la actividad económica que desarrolla. Esta metodología en dos etapas se ha denominado "enfoque autorizado de la OCDE" (Authorized OECD Approach, AOA en adelante) $)^{14}$.

En Colombia, la principal particularidad en la reglamentación de las pautas para asignar beneficios a EP reside en que solo se atribuirán aquellas rentas consideradas de fuente nacional de acuerdo con la definición del concepto contenida en el artículo 24 del Estatuto Tributario, según dispone el artículo 20-2 del mismo cuerpo lega ${ }^{15}$. El gravamen del EP no se basará en la atribución de renta mundial, por tanto, siendo esta una diferencia notable respecto del gravamen de sociedades residentes en el país andino.

El gravamen de rentas empresariales obtenidas a través de un EP se determinará según

\footnotetext{
${ }^{11}$ OCDE (2010). Report on the Attribution of Profits to Permanent Establishments, par. 50; Baker y Collier (2006, p. 26).

${ }^{12}$ Este es el factor que distingue al concepto de entidad funcionalmente separada frente al enfoque del negocio relevante (relevant business approach), que no admite el gravamen de beneficios en sede del EP si la empresa en conjunto ha tenido pérdidas. Vid. OCDE (2010) Report on the Attribution of Profits to Permanent Establishments, par. 8; Burgers (2009, p. 59); Oosterhoff (2008, p. 69); Bennet y Russo (2007, p. 279); Kobetsky (2011, pp. 198-199); Maroto Sáez (2016, p. 462).

${ }^{13}$ Birnkrant (2004,p. 126); Oosterhoff (2008,p. 70); OCDE (2014). BEPS Actions 8, 9 and 10. Draft on Revisions to Chapter I of the Transfer Pricing Guidelines (Including Risk, Recharacterisation, and Special Measures), par. 9 y 11. OCDE (2015). Aligning Transfer Pricing Outcomes with Value Creation, Actions 8-10 - 2015 Final Reports, par. 1.35, 1.36, 1.39, 1.43.

${ }^{14}$ El planteamiento AOA se incorpora por primera vez en los CMCOCDE-2008, si bien su contenido se definió en el informe OCDE (2008). Report on the Attribution of Profits to Permanent Establishments, que a su vez adoptó conceptos concretados en documentos anteriores relativos a la atribución de beneficios a EPs.

${ }^{14}$ El planteamiento AOA se incorpora por primera vez en los CMCOCDE-2008, si bien su contenido se definió en el informe OCDE (2008). Report on the Attribution of Profits to Permanent Establishments, que a su vez adoptó conceptos concretados en documentos anteriores relativos a la atribución de beneficios a EPs.

${ }^{15}$ Vid. Castro Arango (2008, p. 219).
}

Revista de Derecho Fiscal n. ${ }^{\circ}$ • julio-diciembre de 2016 • pp. 43-62 
lo establecido en el artículo 20-2 -según redacción dada por el art.87 de la Ley 1607 de 2012-y 260 y siguientes del Estatuto Tributario -referidos a las reglas de precios de transferencia-, así como por el Decreto 3026 de 2013, que desarrolla tales disposiciones. En dichos preceptos, se adopta un lenguaje idéntico al empleado por parte de la OCDE a la hora de construir el AOA como criterio para la atribución de rentas a EP.

En concreto, el artículo 20-2 señala que las personas naturales no residentes y las personas jurídicas y entidades extranjeras que tengan un establecimiento permanente o una sucursal en el país, según el caso, serán contribuyentes del impuesto sobre la renta y complementarios con respecto a las rentas y ganancias ocasionales de fuente nacional que le sean atribuibles al establecimiento permanente o a la sucursal. Además, la determinación de dichas rentas y ganancias ocasionales se realizará con base en criterios de funciones, activos, riesgos y personal involucrados en la obtención de las mencionadas rentas y ganancias ocasionales. Este mandato encaja por tanto con el contenido de la primera fase del AOA, consistente en atribuir funciones, activos y riesgos para configurar al EP como una entidad funcionalmente separada de su casa central, según hemos tenido ocasión de comprobar en el anterior apartado.

Por su parte, los preceptos referidos al régimen de precios de transferencia colombiano determinan que a efectos de la aplicación de dicha normativa, se consideran vinculados los establecimientos permanentes situados en Colombia y sus casas centrales, de acuerdo con lo dispuesto en el artículo 260.4 del Estatuto Tributario. Aclara además el artículo 260-2 en su párrafo cuarto que "cuando una entidad extranjera, vinculada a un establecimiento permanente en Colombia, concluya una operación con otra entidad extranjera, a favor de dicho establecimiento, este último, está obligado a determinar, para efectos del impuesto sobre la renta y complementarios, sus ingresos ordinarios y extraordinarios, sus costos y deducciones y sus activos y pasivos considerando para esas operaciones el Principio de Plena Competencia".

La coincidencia con el sistema de conceptos que la OCDE plantea se vislumbra con mayor contundencia en lo dispuesto en el artículo 11 del Decreto 3026 de 2012, pues señala que son atribuibles las rentas y ganancias ocasionales que el establecimiento permanente o sucursal habría podido obtener, de acuerdo con el Principio de Plena Competencia, si fuera una empresa separada e independiente de aquella empresa de la que forma parte, ya sea que dichas rentas y ganancias ocasionales provengan de hechos, actos u operaciones entre el establecimiento permanente o sucursal en Colombia con otra empresa, persona natural, sociedad o entidad, o de sus operaciones internas con otras partes de la misma empresa de la que es establecimiento permanente o sucursal.

Si además resultara de aplicación un CDI, habría que atender a lo señalado en el artículo referido a la obtención de beneficios empresariales ${ }^{16}$. Aunque el objeto del presente trabajo no abarca el análisis de

\footnotetext{
${ }^{16}$ En concreto, en el art.7.2 en el MCOCDE-2014 establece que los beneficios imputables son aquellos que el EP hubiese podido obtener, particularmente en sus operaciones con otras partes de la empresa, si fuera una empresa distinta e independiente que realizase actividades idénticas o similares, en las mismas o análogas condiciones, teniendo en cuenta las funciones desarrolladas, los activos utilizados y los riesgos asumidos por la empresa a través del EP y de las restantes partes de la empresa.
} 
la interacción de la normativa interna colombiana con las disposiciones de los CDI suscritos por Colombia, conviene señalar que las reglas de atribución de beneficios a nivel convencional establecen límites cuantitativos a la potestad tributaria del Estado de la fuente y en consecuencia determinan el límite máximo de renta que el Estado de la residencia deberá tener en cuenta a la hora de eliminar la doble imposición. Además, son aplicables de manera autónoma al Derecho interno, pues su cometido es establecer qué rentas ha obtenido un establecimiento permanente a nivel convencional. Por tanto, para conocer el gravamen efectivo de una renta, será necesario aplicar en todo caso las reglas de atribución del Derecho interno del Estado donde el establecimiento permanente se ubique. Si la cuantía atribuible se sitúa por encima del montante calculado según la normativa convencional, la cifra calculada conforme a los parámetros del artículo 7 actuará como límite. Si por el contrario la cuantía es menor, la limitación establecida en el CDI no habrá jugado papel alguno.

\section{Análisis de las reglas de atribución de beneficios a EP en la normativa colombiana}

En el presente apartado analizaremos las reglas de atribución de beneficios a EP en Colombia, al hilo de los criterios que la OCDE establece en relación con la metodología AOA, sobre la que se basa la regulación prevista en el Estatuto Tributario y su normativa de desarrollo.

La determinación de pautas para interpretar la norma interna colombiana es un ejercicio muy necesario, sobre todo si se toma en consideración su actual falta de concreción. Según hemos señalado en el apartado anterior, la renta imputable al EP se determina según las funciones, activos, riesgos y personal que se empleen para su obtención. La mención genérica a tales conceptos provoca el planteamiento de numerosas cuestiones que es necesario resolver. ¿Cuándo podemos considerar que el EP -y no la casa centralestá desarrollando unas funciones concretas? ¿Cuándo se considera que un elemento patrimonial está vinculado funcionalmente a la actividad del EP? ¿Cómo saber si es el EP y no otra entidad quien gestiona un determinado riesgo? Si una serie de trabajadores se desplazan de la jurisdicción donde se sitúa la casa central a aquella donde se encuentra el EP y desempeñan funciones atribuibles al mismo, ¿cómo saber si los trabajadores forman parte del activo del EP o si existiría un acuerdo de cesión de trabajadores entre la casa central y este? ${ }^{17}$.

A continuación procederemos a abordar estos y otros interrogantes con vistas a esclarecer el significado de las reglas establecidas en el Estatuto Tributario para la determinación de las rentas atribuibles al EP. A tal efecto, nuestro análisis partirá de las recomendaciones de la OCDE en la materia, por su calidad técnica y porque son un referente no solo a nivel comparado sino también en el ámbito interno de gran cantidad de Estados con tradiciones jurídicas muy diferentes. Con todo, debemos advertir de nuevo que su valor interpretativo reside únicamente en la fuerza de sus argumentos, no en un pretendido carácter de fuente autorizada, de modo que en ningún caso debería el lector confundir el recurso al contenido de los documentos elaborados por tal institución con

${ }^{17}$ Vid. Sasseville y Vann (2016, p. 57); Calderón Carrero (2015, p. 227) 
su asimilación acrítica ni con la atribución de un valor superior al de cualquier otra fuente de interpretación carente de valor normativo ${ }^{18}$.

\section{Primera fase: conformación de una entidad funcionalmente separada}

En primer lugar, es necesario dotar al EP de funciones, activos y riesgos para lograr la conformación de una ficción funcionalmente separada de la casa central. A continuación analizaremos cada uno de estos factores por separado para comprobar su incidencia en el resultado final.

\subsection{La atribución de funciones como punto de partida del análisis}

El primer elemento a considerar para la concreción del EP como una entidad funcionalmente separada de su casa central son las funciones que el mismo desempeña, concepto que cabe identificar con la realización de actividades y la adopción de responsabilidades sobre una materia determinada ${ }^{19}$. Desde una perspectiva de eficiencia económica, las funciones se deben localizar en la jurisdicción donde se den las condiciones óptimas para ejercitarlas ${ }^{20}$. Por ejemplo, la realización de funciones de fabricación de productos que no requieren conocimientos técnicos complejos para ser desarrolladas se suelen localizar en países donde la mano de obra sea barata para lograr ser competitivo en costes. Sin embargo, la fabricación de productos que requieren la aplicación de conocimientos técnicos complejos o de investigación y desarrollo se llevarán a cabo allá donde haya personal suficientemente cualificado, buena infraestructura, entre $\operatorname{otros}^{21}$.

La identificación de las funciones realizadas por un EP es el primer paso del análisis AOA y probablemente el que más relevancia tiene en el conjunto del planteamiento, porque de este dependerán a su vez la atribución de los activos necesarios para llevarlas a cabo y los riesgos asociados a tales actividades y responsabilidades asumidas ${ }^{22}$. De esta forma será posible atribuir derechos y obligaciones al EP para, en un estadio posterior, determinar las operaciones que llevaría a cabo de haber sido un sujeto independiente, bien sea con la casa central, con entidades vinculadas o con terceros no relacionados. A título de ejemplo, cabe señalar que la atribución de beneficios, ante un supuesto de función de distribución, no será igual si nos encontramos ante un EP distribuidor (full fledged distributor), que normalmente poseerá y gestionará intangibles de marketing para el desarrollo de su actividad, es titular de los inventarios, soporta el riesgo de impago y se hace cargo de la logística, o frente a un EP comisionista (commissonaire), que

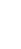


no asume ninguna de las funciones, activos o riesgos señalados ${ }^{23}$.

Hasta la fecha, en el Derecho interno colombiano no se han establecido criterios que concreten la noción de atribución de funciones, al margen de su mención en los distintos preceptos que reglamentan la materia en Colombia. A falta de referencias más adecuadas, los trabajos elaborados por la OCDE en la materia constituyen un buen punto de partida para tratar de dotar de significado a este concepto. En especial, son relevantes tanto el informe de 2010 sobre atribución de beneficios a establecimientos permanentes ${ }^{24}$ como el capítulo I de las TPG10, relativo al análisis funcional de las operaciones entre sujetos vinculados ${ }^{25}$. Dentro de este marco, la OCDE se apoya en el concepto de "funciones humanas sustantivas" (Significant People Functions, SPF en adelante), esto es, en la actividad que el personal desarrolla, para designar las funciones que el EP asu$\mathrm{me}^{26}$. Las SPF son las funciones clave para la generación de beneficios ${ }^{27}$. El predecesor de este criterio es el conocido como criterio KERT (Key Enterpreneurial Risk Taking) centrado en la toma de decisiones efectiva en relación con las actividades de generación de beneficios en que las funciones ejercidas conllevan aparejados activos y riesgos, sin posibilidad de división ${ }^{28}$.

Con todo, resulta extraño que la OCDE seleccione como referente un criterio basado en la realización de funciones a través del personal, pero no especifique pautas para asignar personal a un $\mathrm{EP}^{29}$. La única pauta de referencia en tal sentido determina que las actividades y responsabilidades que se ejerciten en el lugar fijo de negocios que constituye el EP serán atribuibles a este ${ }^{30}$. Con todo, la elección de un criterio basado en la toma de decisiones plantea problemas en tanto las decisiones son procesos mentales que, por ende, no pueden fijarse a un territorio concreto ${ }^{31}$. Dado que desde un EP se pueden ejercer funciones relativas al control del personal, este será también un criterio válido para asignar trabajadores al EP, si bien el personal sin relación laboral será más complicado de asignar en este sentido. Supongamos por ejemplo que la persona encargada de adoptar las decisiones pertinentes que dan lugar a la atribución de funciones al EP es un miembro del consejo de administración que vive en el Estado donde la casa central se sitúa o en otro Estado distinto al

\footnotetext{
${ }^{23}$ Vid. Sasseville y Vann (2016, p. 53).

${ }^{24}$ OCDE (2010). Report on the Attribution of Profits to Permanent Establishments, par.13 et seq.

${ }^{25}$ Vid. TPG-2010 par.1.38-1.63. OCDE (2015) Aligning Transfer Pricing Outcomes with Value Creation, Actions 8-10 - 2015 Final Reports, par. 1.33-1.118.

${ }^{26}$ Vid. Bennet y Russo (2007,p. 280).

${ }^{27}$ Kamphuis (2008, p. 304); Schön (2007, p. 1.065) menciona el principio de causalidad como parámetro de referencia, de tal forma que si el EP lleva a cabo funciones que generen beneficios, éstos se le deberían atribuir.

${ }^{28}$ Kamphuis (2008, p. 304); Schön (2007, p. 1.065) menciona el principio de causalidad como parámetro de referencia, de tal forma que si el EP lleva a cabo funciones que generen beneficios, éstos se le deberían atribuir.

${ }^{29}$ Kroppen, H-K. der "authorized OECD Approach" zur Gewinnaufleitung zwischen Stammhaus und Betriebsstätte /en/ Kessler, W.; Förster, G.; Watrin, C. (eds.) Unternehmenbesteuerung Festschrift für Norbert Herzig zum 65. Geburtstag (Munich: Beck, pp.1071-1084), p.1089, citado en Dziurdz (2014, p. 139); Gómez Jiménez (2009, pp. 134-135).

${ }^{30}$ Vid. OCDE (2010). Report on the Attribution of Profits to Permanent Establishments, par. 60-62.

${ }^{31}$ Schön (2009, p. 103).
} 
de localización del EP. Al establecer que las decisiones se deben adoptar allá donde el EP se sitúa, ¿implicaría ello que dichas funciones en realidad son atribuibles a la casa central? En principio, la solución más adecuada se basaría en conocer si dicha persona efectivamente adopta decisiones relativas a la generación de valor que el EP desarrolla a través de su actividad económica ${ }^{32}$.

En buena parte de los grupos multinacionales existen al menos tres niveles de gerencia, estrechamente relacionados con la implicación temporal de las decisiones que se asumen en cada uno. El nivel más elevado adopta decisiones estratégicas, que afectan al devenir de la empresa a largo plazo, con una amplia capacidad para desarrollar políticas concretas. Los mandos intermedios adoptan decisiones tácticas, a mediano plazo, basadas en las líneas estratégicas marcadas por el nivel superior. También llevan a cabo funciones de coordinación, muy relevantes para lograr una unidad de actuación por parte del conjunto de la empresa. Finalmente, los mandos operacionales adoptan decisiones cuya repercusión tiene lugar en el corto plazo y que supone la concreción de las medidas tomadas por los mandos intermedios ${ }^{33}$.

Según la OCDE, es habitual que la realización de SPF tenga lugar en niveles inferiores al mando estratégico ${ }^{34}$, por lo que se tienen en cuenta decisiones tácticas o de coordinación frente a las decisiones relativas a la fijación de una política empresarial a largo plazo $^{35}$. En este sentido, el establecimiento de límites a la actuación de mandos inferiores, según la OCDE, no implica la toma activa de decisiones.

El empleo del término "sustantivas" implica que el criterio SPF se refiere a aquellas funciones que son clave en la generación de beneficios para la empresa ${ }^{36}$. Por tanto, las funciones complementarias o auxiliares no entrarían dentro de dicha construcción ${ }^{37}$. Se puede concluir que si un EP lleva a cabo funciones auxiliares relativas a una actividad cuyas funciones sustantivas son ejercidas por la casa central, dichas funciones se atribuirán a esta última. Ahora bien, concretar los rasgos diferenciadores entre una categoría y otra no siempre será tarea sencilla en la práctica, puesto que una misma función puede alcanzar distinto nivel de relevancia en un sector de negocio respecto de otro y la estructura de la cadena de valor en cada empresa hace que el análisis deba atender a circunstancias únicas en cada caso ${ }^{38}$. El grado de complejidad aumentará cuando haya que evaluar las funciones calificadas de "relevantes", debiendo jerarquizarlas como más o menos influyentes en la generación de valor para la empresa. Con todo, sí será factible extraer ciertos elementos comunes sobre el alcance del término, sobre todo en lo referido a la distinción entre una serie de categorías fundamentales para la organización estratégica y táctica, como son la planificación, la gestión y el control de actividades y tareas.

Kamphuis señala con acierto que una función se compone al menos de tres elementos:

\footnotetext{
${ }^{32}$ Vid. Schön (2007, p. 1.067).

${ }^{33}$ Vid. Fris, Llinares y Gonnet (2008, p. 5).

${ }^{34}$ OCDE (2008). Report on the Attribution of Profits to Permanent Establishments, par. 28.

${ }^{35}$ Vid. Moerer y Klaver (2015, p. 1.591).

${ }^{36}$ OCDE (2008). Report on the Attribution of Profits to Permanent Establishments, par. 77.

${ }^{37}$ Bernales Soriano (2013, p. 17); Bennett (2008, p. 468).

${ }^{38}$ Oosterhof (2008, pp. 68, 75); Bernales Soriano (2013, p. 17).
} 
el deber de realizar una tarea determinada (responsabilidad), la asunción de poderes de decisión (autoridad) y la existencia de habilidades necesarias para realizar las tareas asignadas, con especial énfasis en los conocimientos requeridos (competencia) ${ }^{39}$. La noción de SPF requiere la concurrencia de estos tres elementos y el referido a la relevancia en la generación de beneficios. La atribución de activos y riesgos, así como la remuneración que llevan aparejada, dependerá de las funciones que cada unidad efectúe. Si a esto unimos la remuneración derivada de la asunción de funciones en sí, habremos completado el análisis AOA propuesto por la OCDE. La correcta determinación de funciones por tanto es crucial, más aún si se tiene en cuenta que las relaciones casa central-EP no se rigen por contratos, por lo que no hay un punto de partida documental en tal sentido, a diferencia de lo que sucede en el ámbito de entidades vinculadas.

La gestión empresarial abarca desde la definición de la estrategia de la empresa (largo plazo), pasando por la asignación o delegación de responsabilidades relativas a la implementación del modelo de negocio (medio plazo), hasta la administración de responsabilidades operacionales (corto plazo $)^{40}$. La asignación racional de poderes de decisión se encuentra ligada al modelo de distribución de competencias en una empresa, que puede fundamentarse en un modelo centralizado o descentralizado, dependiendo de si las decisiones se adoptan en niveles jerárquicos superiores o inferiores. En caso de adopción de un modelo des- centralizado, aumentan las posibilidades de que a un EP se le atribuyan más funciones y viceversa. La decisión sobre la adopción de un modelo centralizado o descentralizado dependerá de diversos factores, aunque el más importante es el referido a la naturaleza específica o genérica de los conocimientos necesarios para poder desempeñar funciones. Los conocimientos específicos abarcan, entre otros factores, la información que requiera acciones inmediatas antes de que pierda valor, información que requiera de interrelaciones con diversos factores para ser aprovechada, información técnica que requiera preparación especializada, información relativa a eventos no predecibles, e información subjetiva o experimental (no cuantificable y complicada de describir) ${ }^{41}$. Los costes de transmitir este tipo de información a instancias jerárquicas superiores dentro de la empresa pueden ser elevados, en cuyo caso la adopción de un modelo descentralizado -en que la capacidad de decisión se sitúa en niveles inferiores- resultará más ajustado. Otros beneficios de este modelo residen en una mayor responsabilidad de mandos intermedios y bajos en el devenir de la empresa a través de la toma de decisiones, así como un alivio en la carga de trabajo del cuerpo directivo de la empresa, si bien a cambio de mayores requerimientos de supervisión y control.

La OCDE se centra únicamente en el proceso de ratificación (toma activa de decisiones) y en el de implementación (gestión), sin otorgar a ninguno de los dos un mayor peso específico. Ello significa que si nos

\footnotetext{
${ }^{39}$ Kamphuis (2008, pp. 301-302). Cfr. OCDE (2008). Report on the Attribution of Profits to Permanent Establishments, par. 118.

${ }^{40}$ Fris, Llinares y Gonnet (2008, p. 7).

${ }^{41}$ Lazear y Gibbs (2006, pp. 100-101).
} 
encontramos ante un supuesto en que la casa central asume el primero y el EP el segundo, o viceversa, las SPF se localizarían en ambos a la vez, con los problemas que ello suscita para completar el resto del análisis $\mathrm{AOA}^{42}$. De hecho, en empresas en que ambos factores se sitúan en niveles jerárquicos distintos, este problema surgirá con frecuencia.

\subsection{La atribución de activos al EP de acuerdo con el concepto de propiedad económica}

La normativa colombiana señala que en el análisis de atribución de rentas a establecimientos permanentes situados en Colombia se deben identificar las funciones "significativas desarrolladas por el personal que sean relevantes para hacer la atribución de la propiedad económica de los activos, así como la atribución misma de la propiedad económica de los activos al establecimiento permanente o sucursal". En este sentido, cabe señalar que la propiedad legal en el marco de la ficción de atribución es irrelevante, ya que el propietario de todos los bienes y derechos es la entidad como unidad indisoluble. Así las cosas, el criterio sobre el que basar la atribución debe necesariamente partir de la noción de propiedad económica, que servirá para asignar al EP aquellos activos necesarios para acometer su actividad ${ }^{43}$. El uso y la gestión del activo en cuestión, tanto desde la perspectiva de su adquisición, como de su desarrollo y posterior venta, serán factores de relevancia en tal sentido ${ }^{44}$. Por tanto, es de suma importancia considerar que si un EP deja de realizar funciones que conducían a la atribución de activos asociados a las mismas, esto ocasionaría la imposición de gravamen por la transmisión de la propiedad económica que se materializaría debido a este cambio de circunstancias ${ }^{45}$.

La atribución de activos al EP puede generar serios problemas si uno considera que el uso de un bien y su incorporación al proceso productivo pueden alcanzarse por medio de distintas vías. Si por ejemplo un EP se dedica al negocio del transporte de pasajeros en vehículos de alta gama, ¿debería atribuirse la titularidad económica de tal inmovilizado al EP o cabría considerar que existe un contrato nocional de leasing entre EP y casa central? ¿Podría articularse el uso a través de un supuesto ficticio de cesión de uso a cambio de un porcentaje de la facturación por la actividad de transporte? La respuesta a estas cuestiones tiene gran relevancia para el cálculo de la renta neta atribuible, en especial en lo relativo al cómputo de gastos de amortización y deterioro en activos tangibles, pagos nacionales de intereses y cánones y atribución de beneficios derivados de la explotación de activos intangibles ${ }^{46}$.

En el ámbito de activos tangibles, el criterio SPF en principio motivaría que la atribución se realizase atendiendo a su gestión desde la perspectiva de su adquisición o fabricación, incorporación al proceso productivo y posterior venta ${ }^{47}$. No obstante, la OCDE considera más apropiado adoptar el criterio del uso del bien, salvo que las

\footnotetext{
${ }^{42}$ Vid. Kamphuis (2008, p. 305).

${ }^{43}$ Vid. Maroto Sáez (2016, p. 467).

${ }^{44}$ Sasseville y Vann (2016, p. 56).

${ }^{45}$ Vid. art.18.5.b), en infra apartado 3.3.6 se trata el contenido de esta cláusula en detalle.

${ }^{46}$ Vid. Van Wanrooij (2004, p. 258).

${ }^{47}$ OCDE (2010). Report on the Attribution of Profits to Permanent Establishments, par. 75.
} 
circunstancias del caso aconsejen optar por otros parámetros. Por tanto, se prefiere dar prioridad a una solución pragmática en vez de mantener la coherencia entre la atribución de funciones señalada y la atribución de activos, si bien cabe señalar que desde la perspectiva del concepto que se maneja de establecimiento permanente como lugar fijo de negocios, resulta adecuado el criterio del uso, por ser menos susceptible de alteración por parte del contribuyente.

Aunque es habitual que el uso y la gestión de un activo coincidan, pueden darse supuestos en los que no sea así. Admitamos que el EP dispone de unas oficinas donde ejercer su actividad, si bien de manera provisional, pues la empresa está tratando de alquilarlo. Desde la casa central se desarrollan todas las funciones relativas a la búsqueda de potenciales arrendatarios, mantenimiento del inmueble, asunción de costes de tasación, etc. En este caso, el EP usa el bien, pero las funciones que viene ejerciendo la casa central apuntan a la atribución del inmueble a esta y necesariamente al reconocimiento de un acuerdo de arrendamiento del inmueble a favor del EP, debiendo abonar a la casa central la renta correspondiente. Con todo, dado que la OCDE emplea el criterio del uso, este supuesto se resolvería a favor de la atribución de las oficinas al EP.

La atribución de los activos intangibles también se basa en la noción de propiedad económica. En términos generales, las funciones que se deben tomar en conside- ración en el ámbito de activos intangibles son aquellas referidas al desarrollo, mejora, mantenimiento, protección y explotación, también conocidas como funciones DEMPE ${ }^{48}$. En todo caso, se plantean problemas adicionales, dada la peculiar naturaleza de este tipo de activos. Los principales atributos que diferencian a los intangibles de los activos tangibles son la posibilidad de explotación por múltiples sujetos sin costes de oportunidad asociados y el hecho de que los retornos de escala aumenten en lugar de disminuir, lo que explica su enorme potencial para generar beneficios.

El hecho de que varios sujetos puedan hacer uso de un intangible al mismo tiempo implica más dificultades a la hora de determinar a quién se debe atribuir el intangible y qué tipo de remuneración debe recibir el propietario $^{49}$ : ¿se debe considerar en todo caso que se concede licencia exclusiva o también cabe entender que podríamos estar ante una licencia no exclusiva? Si nos situamos en el primer caso, ¿la licencia sería exclusiva para ciertos territorios o períodos de tiempo únicamente? ¿Tiene esta discusión sentido si partimos de la idea de que la casa central no se va a ver constreñida en absoluto para utilizar el intangible desde una perspectiva jurídico-privada? La contraprestación por el uso del intangible y la remuneración por realización de funciones que contribuyen a que genere retornos resulta tan importante como la correcta atribución del mismo.

\footnotetext{
${ }^{48}$ DEMPE es el acrónimo de Development, Enhancement, Maintenance, Protection and Explotation, una expresión acuñada en el marco de las acciones 8 a 10 del proyecto BEPS, referidas al ámbito de precios de transferencia. Vid. OCDE (2012). Discussion Draft. Revision of the Special Considerations for Intangibles, par. 48 et seq. OCDE (2013). Revised Discussion Draft on Transfer Pricing Aspects of Intangibles, par.65 et seq. OCDE (2014). BEPS Action 8 Deliverable. Guidance on Transfer Pricing Aspects of Intangibles, par.6.32 et seq. OCDE (2015). Aligning Transfer Pricing Outcomes with Value Creation, Actions 8-10 - 2015 Final Reports, par. 6.32 et seq.

49 Birnkrant (2004, p. 130).
} 
Los criterios de atribución son diferentes para casos en los que el intangible se genera en el seno de la empresa o si es adquirido. En el supuesto relativo a intangibles generados internamente, el criterio que la OCDE maneja es la capacidad de decisión sobre la asunción y gestión de riesgos con respeto al desarrollo del intangible ${ }^{50}$. No obstante, la realización de funciones de desarrollo estrictamente no confiere por sí misma la propiedad del mismo. Piénsese por ejemplo en un supuesto en que un EP acuerda con un centro de I+D el desarrollo de un intangible, de tal forma que el EP asume y controla los riesgos que puedan materializarse en el proceso -contingencias por protección de derechos de propiedad intelectual y/o industrial, pérdidas por diseño de prototipos fallidos, etc. - y supervisa su desarrollo -establece fechas límite y metas a cumplir, control de resultados parciales, etc. ${ }^{51}$-. En tal caso, parece lógico atribuir el intangible al EP y no al centro de $\mathrm{I}+\mathrm{D}$, si bien posteriormente será necesario remunerar de manera adecuada las funciones que este ha ejercido.

Respecto de adquisiciones de intangibles a terceros, el análisis variará en función de si la adquisición se refiere a un intangible plenamente desarrollado o si se encuentra inmerso en una etapa de desarrollo, ya que en este último supuesto a las funciones ya mencionadas se añadirán otras, como la cuestión sobre si resulta más adecuado desarrollar el intangible a nivel interno o adquirirlo, la determinación del valor que el intangible puede aportar al proceso productivo, la supervisión de su explotación o un análisis sobre si conviene descartar su uso o venderlo llegado un punto determinado ${ }^{52}$.

\subsection{Especialidades en la atribución de fuentes de financiación al EP}

La asignación de activos al EP lleva aparejada necesariamente la atribución de las fuentes de financiación de las que se deriva su tenencia. En concreto, el artículo 12.f) del Decreto 3026 de 2013 señala que es necesario atribuir capital al establecimiento permanente o sucursal con base en los activos y riesgos atribuidos al establecimiento permanente o sucursal. Con todo, es importante destacar que no solo parte de los recursos propios, sino también parte del pasivo exigible de la empresa que irá a parar al EP. La atribución de activos supone la atribución de la fuente de financiación de los mismos -capital propio y recursos ajenos- ${ }^{53}$, de tal forma que si la adquisición de un activo se ha financiado con recursos ajenos, será el EP el que soporte la carga financiera. Como es lógico, será necesario determinar si dicha financiación se considera como propia y no da lugar al pago de una contraprestación en forma de gasto financiero o si se considera como financiación ajena y sí resulta necesario que el EP retribuya la financiación. Dependiendo de las circunstancias del caso, el resultado que se alcanzará será distinto.

La metodología que propone la OCDE consiste en la atribución de una cifra de capital libre, denominación que se emplea para de-

\footnotetext{
${ }^{50}$ OCDE (2010). Report on the Attribution of Profits to Permanent Establishments, par. 85; Oosterhoff (2008, p. 70); Dziurdz (2014, p. 151).

${ }^{51}$ OCDE (2010). Report on the Attribution of Profits to Permanent Establishments, par. 88; Bennet y Russo (2007, p. 281); Gómez Jiménez (2009, p. 134).

${ }^{52}$ OCDE (2010). Report on the Attribution of Profits to Permanent Establishments, par. 94.

${ }^{53}$ Maroto Sáez (2016, p. 467).
}

Revista de Derecho Fiscal n. ${ }^{\circ}$ • julio-diciembre de 2016 • pp. 43-62 
signar aquellos recursos que no requieren pagos por concepto de gastos financieros. Una vez realizado el cálculo, el resto de los recursos financieros se consideran ajenos y su deducibilidad quedaría supeditada a la normativa interna de cada Estado.

La OCDE escogió la denominación "capital libre" ${ }^{14}$ para recalcar que las necesidades de financiación de un EP no pueden cubrirse únicamente mediante recursos ajenos que den lugar a pagos deducibles efectuados a la casa central. Según señala la OCDE, de acuerdo con el principio de plena competencia, el EP debe contar con una estructura de capital adecuada y próxima a la que tendría una entidad en sus mismas circunstancias -esto es, tipo de industria, de mercado en que el EP interviene, etc.-, teniendo en cuenta las funciones asumidas, los activos requeridos para realizarlas y los riesgos inherentes a las mismas ${ }^{55}$.

A tal efecto, existen distintos métodos válidos para atribuir capital libre, si bien la elección del más apropiado dependerá de las circunstancias del caso concreto ${ }^{56}$. En concreto, pueden mencionarse al menos tres: 1) atribución de capital de acuerdo con la proporción de activos y riesgos atribuidos al EP, 2) atribución teniendo en cuenta no solo funciones y activos, sino también los riesgos potenciales que puedan materializarse y 3 ) atribución de capital libre por comparación con una entidad independiente que lleva a cabo las actividades similares bajo similares condiciones en el Estado donde el EP se encuentra ${ }^{57}$.

Cabe señalar que existe riesgo de que se produzca doble imposición en aquellos supuestos en que el método empleado por el Estado donde se sitúa el EP y el Estado donde se localiza la casa central no coincide, o coincidiendo, no coincide en mostrar un resultado idéntico ${ }^{58}$. En especial, el tercer método señalado originaría una atribución dispar en función de la admisibilidad en ambos Estados de los comparables propuestos por el contribuyente. La OCDE indica que dicha asimetría debería resolverse a favor del criterio del Estado de la fuente si la diferencia en atribución de capital responde al empleo de criterios que emanan del Derecho interno ${ }^{59} \mathrm{o} \mathrm{si} \mathrm{el} \mathrm{rango} \mathrm{cuantitativo} \mathrm{de} \mathrm{atribu-}$ ción de capital aceptado por el Estado de la fuente es acorde con el principio de plena competencia, independientemente de si el del Estado de la residencia también cumple con este parámetro ${ }^{60}$.

En lo que respecta a la financiación ajena, la OCDE plantea dos métodos distintos para su atribución ${ }^{61}$. Por un lado, se propone el enfoque del trazado (tracing approach), que consiste en analizar las operaciones financieras que representaron una transferencia de recursos de la casa central al EP.

\footnotetext{
${ }^{54}$ CMCOCDE-2014 art.7, par. 21 in fine.

${ }^{55}$ CMCOCDE-2014 art.7, versión pre-2010, par. 45. Bennet, M.; Russo, R. (2009), p. 78.

${ }^{56}$ CMCOCDE-2014 art.7, versión pre-2010, par. 46.

${ }^{57}$ Los nombres en inglés son: (1) Capital Allocation Approach (2) Economic Capital Allocation Approach (3) Thin Capitalization Approach. Vid. Burgers (2009, p. 63).

${ }^{58}$ Vid. Baker y Collier (2009, p. 202).

${ }^{59}$ En línea con lo señalado en los CMCOCDE14 art. 23, par. 32.1 et seq.

${ }^{60}$ CMCOCDE-2014 art.7, versión pre-2010, par. 48.

${ }^{61}$ OCDE (2010). Report on the Attribution of Profits to Permanent Establishments, par.152 et seq.; Vid. Bakker y Van Hoey Smith (2004, p. 18).
} 
Por otro lado, el enfoque de la fungibilidad (fungibility approach) parte de la base de que toda la deuda de la entidad contribuye a la financiación ajena del EP, de forma que para el cálculo de la deuda atribuible al EP se adoptaría como referencia que ligue el empleo de recursos ajenos a la actividad económica (activos atribuidos, gastos de personal, entre otros) ${ }^{62}$.

En última instancia, serán las normas de cada Estado las que determinarán si el pago de intereses por parte del EP computa como gasto deducible o si existen restricciones al respecto. En Colombia será necesario atender a la reglamentación en la materia según lo dispuesto en el Estatuto Tributario en lo referido al impuesto sobre la renta y disposiciones de desarrollo de su contenido.

\subsection{La asignación de riesgos al EP como entidad funcionalmente separada}

De acuerdo con lo dispuesto en el artículo 12.c), se atribuirán al EP los riesgos que asuma de acuerdo con las funciones realizadas. En tal sentido, es la toma activa de decisiones relativa a la gestión diaria la que constituye el verdadero criterio de atribución, que a su vez dependerá en gran medida de la naturaleza del riesgo ${ }^{63}$.

En caso de que exista una disociación entre funciones y activos, resulta preceptivo efectuar un análisis sobre su atribución y la posible compensación que se requiera para reflejar qué harían partes independientes en circunstancias similares. Esto conduciría a plantearse si existen ciertas funciones ligadas exclusivamente a gestionar o mitigar riesgos. Estos matices justifican el hecho de que se puedan externalizar ciertas funciones ligadas a los riesgos sin que se produzca una traslación de los mismos ${ }^{64}$. Imaginemos que un EP se dedica a la fabricación y distribución de productos, tarea que habitualmente lleva asociada la realización de funciones tendentes a la disminución del riesgo por devolución del producto. Supongamos sin embargo que el personal de la casa central es el que realiza controles de calidad, imparte cursos a los operarios para disminuir la tasa de error en la fabricación de cada componente del producto final y realiza labores de supervisión sobre los inputs adquiridos para fabricar el producto. En tal caso, si bien el EP fabrica y distribuye el producto, asumiendo en principio el riesgo económico de pérdidas por productos defectuosos, en este caso la realización de funciones por parte de la casa central tendentes a mitigar el mismo provoca que el riesgo sea atribuible a esta y no al EP, que deberá compensar a la casa central por la labor realizada.

No conviene olvidar que en el contexto de las relaciones entre un EP y su sede central, es el conjunto de la empresa el que asume el riesgo, desde una perspectiva jurídica ${ }^{65}$. Es por este motivo que dada la naturaleza de la ficción que el artículo 7.2 impone, el criterio de capacidad financiera para soportar el riesgo, que es otro de los criterios que

\footnotetext{
${ }^{62}$ Sasseville y Vann (2016, p. 59); Gómez Jiménez (2009, p. 145).

${ }^{63}$ OCDE (2010). Report on the Attribution of Profits to Permanent Establishments, par. 22; Moerer y Klaver (2015, p. 1.592).

${ }^{64}$ OCDE (2010). Report on the Attribution of Profits to Permanent Establishments, par. 70; Dziurdz (2014, p. 151); Bennett (2008, p. 468).

${ }^{65}$ Gómez Jiménez (2009, p. 138).
} 
es usual emplear en el ámbito de atribución de riesgos ${ }^{66}$, es irrelevante en el ámbito de la conformación del EP como entidad funcionalmente separada. Será la entidad en conjunto la que deba asumir posibles consecuencias negativas y por tanto la que deba disponer de medios adecuados al efecto. De no ser así, cabría afirmar que, en realidad, la entidad no está asumiendo el riesgo en cuestión. En este sentido, las personas que adoptan las decisiones relativas a la asunción y gestión de riesgos no soporten las pérdidas derivadas de su materialización, por lo que se genera una asimetría difícilmente salvable, que puede dar lugar a una atribución arbitraria de beneficios.

\section{Segunda fase: atribución de ingresos y gastos derivados de acuerdos entre la casa central y el EP, así como con entidades terceras}

Una vez se ha completado el primer paso del AOA, consistente en dotar al EP de una entidad propia y separada de la casa central, la segunda parte del análisis requerirá analizar las operaciones internas (dealings) que se hubieran celebrado entre ambos de haber sido partes independientes conforme al principio de plena competencia, según determinan las letras a) y e) del artículo 12 del Decreto
3026 de 2013. Su valoración se efectuará de manera idéntica a las transacciones efectuadas entre entidades independientes que lleven a cabo funciones similares, empleen activos parecidos y asuman riesgos semejantes y que posean las mismas o similares características económicas relevantes ${ }^{67}$.

Según establecen los informes emitidos por la OCDE, el punto de partida para considerar la existencia de acuerdos será la contabilidad del EP y la revisión de documentación interna de la empresa ${ }^{68}$. De hecho, se recomienda mantener soporte documental sobre el que se plasmen los acuerdos efectuados entre casa central y EP para facilitar el análisis de atribución de beneficios ${ }^{69}$, en forma de documentos contables, emisión de facturas proforma o acuerdos cuasicontractuales ${ }^{70}$. No obstante, antes de reconocer la existencia de un acuerdo, uno debe cerciorarse de que este sería susceptible de ser celebrado entre partes independientes ${ }^{71}$.

El resultado del primer estadio, esto es, de la conformación del EP como ente distinto y separado de la casa central, con funciones, activos y riesgos atribuidos, es de gran relevancia en este segundo estadio. Si por ejemplo un intangible ha sido atribuido a la casa central, pero el EP a su vez lleva a cabo funciones DEMPE, la casa central remunerará dicha actividad en consecuencia. La elección

\footnotetext{
${ }^{66}$ Vid. TPG-2010, par. 9.29-9.32. OCDE (2014). BEPS Actions 8, 9 and 10. Draft on Revisions to Chapter I of the Transfer Pricing Guidelines (Including Risk, Recharacterisation, And Special Measures), par. 66. OCDE (2015). Aligning Transfer Pricing Outcomes with Value Creation, Actions 8-10 - 2015 Final Reports, par. 1.64.

${ }^{67}$ OCDE (2010). Report on the Attribution of Profits to Permanent Establishments, par. 17.

${ }^{68}$ CMCOCDE-2014 art. 7 par. 213. Vid. Baker y Collier (2006, p. 41), que señalan que prácticamente todos los informes de países elaborados en el marco de la IFA 2006 indican que la contabilidad es el punto de partida para la atribución de beneficios a un EP.

${ }^{69}$ OCDE (2010). Report on the Attribution of Profits to Permanent Establishments, par. 36. Vid. Van Wanrooij (2004, p. 253).

${ }^{70}$ Dziurdz (2014, p. 143).

${ }^{71}$ OCDE (2010). Report on the Attribution of Profits to Permanent Establishments, par. 35
} 
del método aplicable será crítica para saber qué porción del beneficio residual permanecerá en la casa central y cuál irá a parar al EP vía remuneración ${ }^{72}$.

A continuación proponemos un ejemplo que permite vislumbrar los problemas a la hora de determinar la existencia de un acuerdo y la naturaleza que se le debe atribuir: imaginemos que un EP fabricante elabora un producto por encargo del dueño de un intangible cuya explotación es necesaria para la producción, de tal forma que se permite que el EP lo use sin coste alguno, porque su labor de fabricación es en beneficio del dueño del intangible. Si por el contrario, el EP fabricase el producto en su propio beneficio, no podría utilizar el intangible de manera gratuita y se vería constreñido a pagar regalías. En el ámbito de la metodología AOA, ¿cuál de estas dos opciones debería tomarse como válida? $?^{73}$. En primer lugar, sería necesario determinar quién es el propietario económico del intangible de acuerdo con las pautas señaladas más arriba. Supongamos que, para un mayor desarrollo del ejemplo, el titular económico coincide con el jurídico; en este caso, la casa central. Seguidamente, habría que determinar qué hubiesen acordado partes independientes en situaciones similares, a través de un estudio basado en el análisis de comparabilidad según la metodología establecida en la normativa colombiana, basada en las guías sobre precios de transferencia elaboradas por la OCDE.

\section{La relevancia de la contabilidad en la atribución de rentas a $\mathrm{EP}$}

Una vez finalizado el análisis de las pautas de atribución de rentas, conviene realizar una serie de consideraciones al hilo de la importancia de su correcto reflejo documental. El artículo 20-2 del Estatuto Tributario señala que para propósitos de la atribución de las rentas y ganancias ocasionales a que se refiere este artículo, los establecimientos permanentes y las sucursales de sociedades extranjeras deberán llevar contabilidad separada en la que se discriminen claramente los ingresos, costos y gastos que les sean atribuibles. Además, la contabilidad de los establecimientos permanentes y de las sucursales de sociedades extranjeras deberá estar soportada en un estudio sobre las funciones, activos, riesgos y personal involucrados en la obtención de las rentas y de las ganancias ocasionales atribuidos a ellos.

El artículo 13 del Decreto 3026 de 2013 incide en este particular al señalar que la contabilidad debe estar debidamente soportada en el estudio al que se refiere el artículo anterior y en los documentos que sirvan de soporte externo de las operaciones, actos y hechos registrados en la misma. En dicha contabilidad deberán registrarse las rentas y ganancias ocasionales atribuidas a los establecimientos permanentes y sucursal que provengan de operaciones realizadas tanto con empresas independientes como con 
empresas vinculadas, así como las relativas a operaciones internas, tales como el reconocimiento de capital, pasivos, derechos, obligaciones, la atribución de la propiedad económica de activos usados por el establecimiento permanente con su consecuente depreciación o amortización cuando haya lugar a ello.

Así las cosas, la normativa señala con especial énfasis que la contabilidad se encuentra supeditada a un análisis correcto de los hechos del supuesto, de manera que el análisis de la atribución de funciones, activos y riesgos que el AOA exige resulta apropiado para corroborar si la contabilidad refleja de manera adecuada la base fáctica de un caso concreto ${ }^{74}$. De otra parte, la contabilidad constituye un documento valioso para valorar si una entidad ha aplicado correctamente el AOA o si existe una desviación entre ambos. Así, en caso de que la documentación que ha preparado el contribuyente refleje la realidad económica de la atribución de activos, funciones y riesgos al EP y los acuerdos que este ha alcanzado con la casa central y otros sujetos, tal asignación debería respetarse ${ }^{75}$. En tal sentido, contabilidad y análisis funcional deben coincidir. De no ser así, como es lógico, la realidad debe primar sobre un reflejo documental erróneo de la misma. No son por tanto conceptos contrapuestos, sino que se complementan entre sí.

\section{Conclusiones}

En el presente trabajo se han analizado las reglas para la determinación de la renta de establecimientos permanentes situados en Colombia. A tal efecto, se ha llevado a cabo un estudio sobre la base de las pautas que la OCDE establece en la materia, ya que el sistema de conceptos propuesto por este organismo internacional se adoptó como referencia por parte del legislador colombiano a partir de 2012 .

El análisis de la atribución de rentas se debe llevar a cabo en dos fases. En la primera, se atribuyen funciones, activos y riesgos para concretar la ficción de entidad funcionalmente separada sobre la que se basa el EP. En una segunda fase, se concretan los acuerdos y operaciones internas que el EP acomete con su casa central, así como las operaciones que desarrolle con entidades de grupo o con terceros independientes. En tal sentido, la complejidad de la materia y la escasez de pautas existente en el Derecho colombiano en la actualidad son dos factores de peso para que el legislador se plantee positivizar reglas que concreten el mandato de atribución contenido en el Estatuto Tributario.

\footnotetext{
${ }^{74}$ En el ámbito de precios de transferencia, la importancia de la documentación para la conformación de los hechos, que si bien no es determinante es de importancia para tener un punto de partida sólido. Vid. TPG-2010 par.1.52-1.53. OCDE (2014). BEPS Actions 8, 9 and 10. Draft on Revisions to Chapter I of the Transfer Pricing Guidelines (Including Risk, Recharacterisation, and Special Measures), par. 2, 3. OCDE (2015). Aligning Transfer Pricing Outcomes with Value Creation, Actions 8-10 - 2015 Final Reports, par. 1.42-1.43.

${ }^{75}$ Birnkrant (2004, p. 127). Como vemos, la diferencia de la importancia de la documentación entre las relaciones matriz-filial y casa central-EP no es tan distante como pueda parecer en un primer momento. Cfr. Dziurdz (2014, p. 142).
} 


\section{Referencias}

\section{Bibliográficas}

Baker, P.; Collier, R. (2006). "General report", en The Attribution of Profits to Permanent Establishments (IFA Cahiers de droit fiscal international).

Baker, P.; Collier, R. (2009). "2008 OECD Model: Changes to the Commentary on Article 7 and the Attribution of Profits to Permanent Establishments", en Bulletin for International Taxation, vol.63, n.5/6, p.199-203).

Bakker, A.; Van Hoey Smith, A. (2004). "Recent Developments Regarding Deductibility of Interest on Loans Provided by a General Enterprise to its Permanent Establishment", en International Transfer Pricing Journal, vol.11, n.1, p.13-19).

Bennet, M. (2008). "The Attribution of Profits to Permanent Establishments: The 2008 Commentary on Art. 7 of the OECD Model Convention", en European Taxation, vol.46, n.1, p.467-471).

Bennett, M.; Russo, R. (2007). "OECD Project on Attribution of Profits to Permanent Establishments: An Update", en International Transfer Pricing Journal, vol.14, n.5, p.279-284).

Bennet, M.; Russo, R. (2009). "Discussion Draft on a New Art. 7 of the OECD Model Convention”, en International Transfer Pricing Journal, vol.16, n.2, p.73-80).

Bernales Soriano, R. (2013). “The Authorized OECD Approach: An Overview”, en Gutiérrez, C.; Perdelwitz, A. (eds.) Taxation of Business Profits in the 21st Century. Amsterdam: IBFD, p. 135-181).

Birnkrant, H. (2004). "Through the Looking Glass: the OECD Discussion Draft Treatment of a Permanent Establishment as a Functionally Separate Entity", en Tax Management Memorandum.

Black, A. (2010). “Attribution of Profits to PEs - Implications of the 'Authorized' OECD Approach (Part 1) ”, en Journal of International Taxation, Vol. 21, nº. 2, pp.19-29, 62-64.

Burgers, I. (2009). "The New OECD Approach on Profit Allocation: A Step Towards Neutral Treatment of Permanent Establishments and Subsidiares", en Florida Tax Review, vol.10, n.1, p.51-76).

Calderón Carrero, J.M. (2015). "Beneficios empresariales (y de navegación) ”, en Carmona Fernández, N. (coord.) Convenios fiscales internacionales y fiscalidad de la Unión Europea. Valencia: CISs.

Castro Arango, J.M. (2008). "El establecimiento permanente. Primicias y problemáticas en Colombia", en Revista de Derecho Fiscal, vol. 4, pp.195-226.

Dziurdz, K. (2014). "Attribution of Functions and Pro ts to a Dependent Agent PE: Different Arm's Length Principles under Articles 7(2) and 9?”, en World Tax Journal, vol.6, n.2, pp. 135-167.

Escribano López, E. (2015). "An Opportunistic, and yet Appropriate, Revision of the Source Threshold for the Twenty-First Century Tax Treaties", en Intertax, vol. 41, n. 1, pp. 6-13.

Fris, P.; Llinares, E.; Gonnet, S. (2008). "PEs and transfer pricing: the playing field in international taxation redefined", en Tax Planning International Transfer Pricing, vol. 12, pp.1-6.

García Prats, F.A. (1996). El establecimiento permanente. Análisis jurídico-tributario internacional de la imposición societaria. Madrid: Tecnos.

Gómez Jiménez, I. (2009). "Un análisis sobre el enfoque autorizado de la OCDE para la atribución de beneficios a establecimientos permanentes", en Crónica Tributaria, na. 133, pp. 125-156. 
Kamphuis, E. (2008). "Signifincant People Functions and Funcional Ownership: The New Motto in Transfer Pricing”, en Tax management transfer pricing report, vol. 17, n . 7, pp. 300-310.

Kobetsky, M. (2011). International Taxation of Permanent Establishments. Cambridge: Cambridge University Press.

Lazear, E.; Gibbs, M. (2006). Managing the Workplace. Chicago: University of Chicago Press.

Malherbe, J.; Daenen, P. (2010). "Permanent Establishments Claim Their Share of Profits: Does the Taxman Agree?", p. 361.

Maroto Sáez, A. (2016) “Atribución de beneficios a establecimientos permanentes. Operaciones vinculadas”, en Carmona Fernández, N. (dir.) Nuevo régimen fiscal de las operaciones vinculadas. Valencia: CISS.

Moerer, O.; Klaver, B. (2015). “The AOA, PE status and BEPS, some reassurance in a time of change?”, en Transfer pricing international journal, vol. $16, \mathrm{n}^{\circ} .4$.

Oosterhoff, D. (2008). “The True Importance of Significant People Functions”, en International Transfer Pricing Journal, vol. 15, n. 2, pp. 68-75.

Pijl, H. (2006). "The OECD Commentary as a Source of International Law and the Role of the Judiciary", en European Taxation, vol. $46, \mathrm{n}^{\circ} .5$, pp. 216-224.

Sasseville, J.; Vann, R. (2016). “Article 7: Business Profits”, en Global Tax Treaty Commentaries. IBFD Database, último acceso a 10 de enero de 2016).

Schön, W. (2007). "Attribution of Profits to PEs and the OECD 2006 Report”, en Tax Notes International, vol. $46, \mathrm{n}^{\circ} .10$, pp. 1.059-1.072.

Schön, W. (2009). "International Tax Coordination for a Second-Best World (Part I)", en World Tax Journal, vol. $1, \mathrm{n}^{\circ} .1$, pp.67-114.

Skaar, A. (1991). Permanent Establishment. Erosion of a Tax Treaty Principle. Deventer: Kluwer Law and Taxation Publishers.

Van Wanrooij, J. (2004). "Attribution of profits to a permanent establishment: the OECD Model, the Working Hypothesis and the Dutch approach”, en British Tax Review, n. 3, pp. 248-280.

Vann, R. (2006). “Tax Treaties: The Secret Agent’s Secrets”, en British Tax Review, n’. 3, pp. 345-382.

Yong, S. Y. (2012). “Tax Optimization Using Branches? ”, en Bulletin for International Taxation, vol. 66, n. 8, pp. 424-435.

Zornoza Pérez, J.J. (2010). "La problemática expansión del Soft Law: un análisis desde el derecho tributario", en Revista de Tributación. Asociación Argentina de Estudios Fiscales, nº. 30, pp. 51-75. 